\title{
Annotations
}

\section{Chorion villus biopsy}

The standard method for obtaining fetal tissue is by means of amniocentesis. Amniocentesis is usually performed at about the 16th week of gestation because the ratio of viable to non-viable cells is inadequate before this time, and culture requires another three to four weeks. The typical patient will therefore be in the middle of the second trimester before the result is available. The other classic techniques for obtaining fetal tissue, such as fetoscopic or ultrasound directed fetal blood sampling, liver biopsy, and skin biopsy, are also carried out in the middle of the second trimester. By this time, the mother will be visibly pregnant and aware of her baby's movements. Termination of pregnancy, if it is required, is not only more dangerous and painful, but is associated with much more severe emotional problems than first trimester termination. It is not surprising, therefore, that chorion biopsy, which can provide a definitive diagnosis at 10 weeks of gestation, has attracted immense attention from both within and outside the medical profession. The possibility of harvesting chorionic tissue has been known for two decades, but current interest was generated by a simple technique of transcervical villus aspiration developed in the Tietung hospital of an iron and steel company in mainland China. ${ }^{1}$ This technique was not ultrasound guided, and the authors (whose names are not mentioned, in keeping with the egalitarian principles of their country) claimed a very high success rate and an abortion rate of only $5 \%$ of continuing pregnancies. The next report of transcervical villus aspiration technique comes from the Soviet Union by Kazy, ${ }^{2}$ and subsequently the technique was introduced to the west by Mr Frank Loeffler, a consultant at St Mary's Hospital in London. This technique was initially carried out without ultrasound control but it was soon found that this was necessary for clinical use and all serious investigators throughout the world now use an ultrasound directed technique. Over the past two and a half years, since the first report in the west from St Mary's Hospital, the technique has been applied widely in Britain, Italy, Scandinavia, Denmark, the Netherlands, France, and the United States. A central registry has been formed in Philadelphia and over 4000 cases have been reported to this source. The most recent refinement of this technique has been the development of a transabdominal method for obtaining chorionic villus tissue. $^{3-5}$

\section{Timing of chorion villus biopsy}

Most workers feel that it is undesirable to perform a chorion villus biopsy before eight weeks of gestation because the chorion frondosum is not clearly identifiable before this. It is widely regarded that the optimal time for transcervical chorion villus biopsy is between eight and 11 weeks of gestation. The transabdominal technique may, however, be carried out beyond this time.

\section{Technique of transcervical chorion villus biopsy}

Current techniques for transcervical chorion biopsy may be grouped into those dependent on hysteroscopy and biopsy forceps and the more widely used techniques of aspiration through a malleable cannula. The placental site is identified by means of the ultrasound machine, with the operator's fingers in the vagina so that the exact relation to the cervix can be ascertained. If a cannula is to be used it is bent into the appropriate shape and inserted through the cervix and guided into the chorion fondosum. The aspiration is normally taken about half way between the edge of the forming placenta and the umbilical insertion and the aspirate is immediately examined in theatre. ${ }^{6}$

\section{Technique of transabdominal chorion biopsy}

The fundus of the pregnant uterus comes to lie against the abdominal wall after about nine weeks of gestation. The uterus can therefore be reached through the abdominal wall at this point without transversing the bladder or bowel. This route provides access to both the anterior and posterior walls of the placenta, but, in order to reach the latter without penetrating the amniotic cavity, an empty bladder is required. (In very rare cases, usually involving grand multiparous patients, the uterus may not be sufficiently anteverted at this stage of pregnancy and we have developed a perurethral, transvesical technique for this situation). An $18 \mathrm{G}$ 
stillete pointed needle is inserted through the abdominal and uterine walls to reach the placental edge. This provides a conduit for a thinner $20 \mathrm{G}$ needle which is inserted to a point midway between the placental edge and the umbilical cord insertion. It is from here that villi are aspirated and, if the first attempt is unsuccessful (which is rarely the case), the outer needle can again serve to guide the aspirating needle to the correct position. In this way, the need for multiple insertions through the uterine wall is obviated.

\section{Analysis of chorion villus tissue}

The most frequent indication for chorion villus sampling is for karyotyping, but gene probe analysis, for which chorion tissue is particularly suitable, is a rapidly growing indication. Admittedly this technique can be applied to cultured amniotic fluid cells which provide just enough DNA for the technique; however, the large number of cells in chorionic villi provide sufficient DNA for direct processing without the need for culture. Enzyme analysis for a number of inborn errors of metabolism can also be carried out on chorion.

Samples may be prepared for cytogenetic analysis in one of two ways. The first is conventional culture. For this villi must be cut into very thin slices to expose mesenchymal cores. ${ }^{7}$ Fibroblasts from this area proliferate under culture conditions and provide suitable chromosomes for analysis.

The so called direct preparation chromosome analysis relies on the fact that a large number of cells in the chorion fondosum will be in the process of division at any one time. These cells are therefore more amenable to analysis without culture. ${ }^{8}$ One advantage of direct preparation is that the result is available to the patient within hours. An even greater advantage is that this method effectively eliminates concern over the possibility of maternal contamination. The main disadvantage of the direct method is that the chromosomes are usually not as elongated and clearly separated as those obtained from cultured cells and impeccable banding is therefore more difficult to achieve. Most laboratories therefore rely on direct preparation to provide a rapid answer regarding fetal sex or aneuploidy and culture to provide information about minor deletions and rearrangements. A theoretical concern has been the possibility that the chromosomal constitution of the trophoblast may not reflect that of the fetus itself. The formation of a mosaic in the early embryo may result in a normal cell line in the embryonic disc with the cells containing the abnormal chromosome complement confined to the trophoblast. It is interesting to note, therefore, that a number of cases have now been described where trisomy of chromosome 16 was detected on chorion biopsy. This is a lethal malformation if present in the embryo and subsequent amniocentesis in these cases has, not surprisingly, shown that the fetus itself was unaffected. The trophoblast can apparently function normally in the presence of this trisomy.

About 15 or $20 \mathrm{mg}$ of chorionic tissue is required to extract sufficient DNA (approximately $20 \mu \mathrm{g}$ ) to perform gene probe diagnosis. This methodology has, to date, been most widely used for the diagnosis of haemoglobinopathies. ${ }^{9}$ Gene probes, however, are available for a number of other conditions such as Duchenne muscular dystrophy, haemophilia, Christmas disease, Huntington's chorea, ornithine carbamyl transferase deficiency, and many others. The technical details on which gene probe analysis depend are beyond the scope of this article. Here it is sufficient to point out that there are two kinds of gene probe diagnosis. ${ }^{1011}$ The first and easiest occurs when the gene is completely deleted (as in alpha thalassaemia) or the aberrant mutation removes a cutting site for the endonuclease enzyme which is used to chop DNA into many small fragments (this is the case in sickle cell anaemia). For most conditions, however, this kind of diagnosis is not possible and linkage studies, to the presence or absence of various endonuclease cutting sites in the DNA which separates genes, are required.

A growing number of inborn errors of metabolism can now be diagnosed by means of enzyme assay on chorion tissue. Most of these belong to the lysosomal storage diseases. The analysis may be carried out on cultured cells or directly from uncultured chorionic villi.

\section{Safety of chorion villus biopsy}

The most striking feature of the Philadelphia register is the wide variation in reported abortion rates for transcervical chorion biopsy, with figures varying from $3 \%$ to $30 \%$. The half dozen European and American centres with the greatest experience report an abortion rate of approximately $4.5 \%$. The background abortion rate at this stage of pregnancy, given an intact fetus on ultrasound, is about $1.5 \%$. The procedure related abortion rate in these eminent centres is therefore approximately $3 \%$. Much higher abortion rates have been reported from British centres. Abortions follow two patterns. A proportion of the abortions occur within the first week of sampling and manifest with bleeding followed by abortion. A second and equally common form of abortion occurs between one and five weeks after the procedure. The fetus grows initially but this is followed by severe oligohydramnios, loss 
of the fetal heart and then abortion. The first pattern can be ascribed to mechanical disruption (subchorial haematoma) and this is rarely seen in units with considerable experience. The second pattern of abortion, however, is presumed to be due to infection, possibly of chronic form, with an organism such as listeria or chlamydia. In addition, four cases of septic shock (one requiring hysterectomy) have followed transcervical chorion villus biopsy. Only two series of transabdominal chorion biopsy have been reported to date. These provide the lowest abortion rates yet reported. Transabdominal biopsy avoids the contaminated cervical area and this should greatly reduce the chances of infection. We know from experience with amniocentesis that the skin is bacteriologically sound. Many patients prefer this technique to the vaginal manipulation and lithotomy position which are necessary for transcervical biopsy.

\section{Future developments}

Ultrasound guided invasive techniques are having a major impact in many branches of medicine and transabdominal chorion biopsy may be regarded as an example of this trend. It will, however, be important to follow up children on whom this technique has been employed in order to detect any as yet unseen complications. The most important development in the future is likely to be the increasing use of gene probe technology which will allow the diagnosis of a very large number of inherited conditions. Further in the future the possibility of analysing trophoblast cells separated from maternal serum may be achieved. The development of a probe for the 21 chromosome and in situ hybridisation techniques may lead to a blood test for Down's syndrome. The possibility of gene therapy in utero or in the early embryo should be mentioned; however, our poor understanding of the regulation of gene control precludes any immediate development of this possibility.

\section{References}

1 Tietung Hospital of Anshan Iron and Steel Company. Fetal sex prediction by sex chromatin of chorionic villi cells during early pregnancy. Chin Med J [Engl] 1975;1:118-25.

2 Kazy Z, Rovosky IS, Bakharev VA. Chorion biopsy in early pregnancy: a method of carly prenatal diagnosis for inherited disorders. Prenat Diagn 1982;2:39-45.

${ }^{3}$ Lilford RJ. Chorion villus biopsy. Maternal and Child Health 1985:10:198-202.

${ }^{4}$ Lilford RJ, Maxwell D. The development of a transcutancous technique for chorion biopsy. Prenatal Diagnosis Group Newsletter 1984;8:4-10.

5 Smidt-Jensen S, Hahnemann N. Transabdominal fine needle biopsy from chorionic villi in the first trimester. Prenat Diagn 1984:4:163-9.

${ }^{6}$ Maxwell D, Czepulkowski BH, Heaton DE, Coleman DV, Lilford RJ. A practical assessment of ultrasound-guided transcervical aspiration of chorionic villi and subsequent chromosomal analysis. Br J Obstet Gynaecol 1985;92:660-5.

${ }^{7}$ Niazi M, Coleman DV, Loeffler FE. Trophoblast sampling in early pregnancy. Culture of rapidly dividing cells from immature placental villi. Br J Obstet Gynaecol 1981;88:1081-5.

* Simoni G. Brambati B. Danesino C, et al. Efficient direct chromosome analyses and enzyme determinations from chorionic villi samples in the first trimester of pregnancy. Hum Genet 1983;63:349-57.

" Maxwell D, Lilford R, Morsman J, Rodeck C, Old J, Thein S. Direct DNA analysis for diagnosing fetal sickle status in first trimester chorion tissue. J Obstet Gynaecol 1985;5:133-5.

11) Williamson R. Thalassacmia: from theory to practice. Nature 1981:292:405-6.

"Williamson R. Eskdale J, Coleman DV, Niazi M, Loeffler FE. Modell BM. Direct gene analysis of chorionic villi: a possible technique for first trimester antenatal diagnosis of hacmoglobinopathics. Lancet 1981;ii:1125-7.

R J LILFORD

Department of Obstetrics and Gynaecology,

St James's University Hospital, Leeds LS9 7IF 